\title{
Mots et sens du territoire dans les démarches d'innovation sociale en Basse-Normandie
}

\author{
Words and meanings associated to the territory \\ in the process of social innovation \\ in Basse-Normandie
}

\author{
Cécile Le Corroller
}

Maître de Conférences

Chercheur associé au CREM - Centre de Recherche en Économie et Management UMR CNRS 6211

Université de Caen Basse-Normandie, UFR des Sciences économiques et de Gestion

Campus 4 - 19 rue Claude Bloch, 14075 Caen

\section{Résumé}

Les entreprises au sens large, d'organisations de production, sont actrices de l'innovation sociale et du développement territorial. Elles agissent de manière plus ou moins coordonnée avec les autres acteurs du territoire et le partage d'un langage commun est un déterminant important de l'action collective. À partir d'une revue de littérature pluridisciplinaire sur le territoire et l'innovation sociale et sur la base de l'analyse textuelle de 50 entretiens réalisés auprès de responsables d'entreprises considérées comme socialement innovantes, un vocable spécifique apparaît.

Aux trois étapes du processus d'innovation sociale correspondent trois conceptions du territoire. Le « territoire interprété » par l'individu pour l'émergence des idées d'innovation sociale, le « territoire construit » collectivement pour la concrétisation et le «territoire institué » ou qui s'institutionnalise pour la diffusion. Le langage commun apparaît plus présent, plus facile à mettre en place, sur la troisième étape que sur les deux premières.

(C) 2012 Lavoisier, Paris. Tous droits réservés.

\footnotetext{
*Adresse email : cecile.lecorroller@ unicaen.fr doi:10.3166/ges.14.287-302 @ 2012 Lavoisier, Paris. Tous droits réservés.
} 


\section{Summary}

Companies, in the broad sense of productive organisations, are actors of social innovation and of territorial development. They act more or less in coordination with the others actors of the territory and sharing a common language is a determinant of collective action.

From a multidisciplinary review of literature on territory and social innovation and from the textual content analysis of 50 interviews with managers of firms considered as socially innovative, a special language appears.

One conception of territory is associated to each three stages of the process of social innovation: an individualy "interpreted territory" for the emergence of idea, a collectively "constructed territory" for concretization and an "institutionalized territory" for diffusion. The common language appears more present and easier to implement, on the third step than on the others.

(C) 2012 Lavoisier, Paris. Tous droits réservés.

Mots clés : Innovation sociale, territoire, local, proximité, développement, analyse textuelle.

Keywords: Social innovation, territory, local, proximity, development, textual analysis.

\section{Introduction}

Le sujet de la recherche présentée dans cet article envisage l'innovation sociale par les entreprises comme des manières de produire autrement, de produire en cohérence avec une vision du monde. L'entreprise devient alors actrice du développement local au même titre que les pouvoirs publics, ce qui suscite des questionnements quant à la nécessité, à la capacité des acteurs à trouver un langage commun, un accord sur les définitions, sur les sens, pour construire un projet territorial commun. «Les innovations sociales ont toujours besoin d'un support, ne serait-ce que celui du langage. »(Lévesque, 2007, p. 55).

Les entreprises ont un rôle dans la société qui même s'il est vu au sens le plus restrictif de «faire du profit » inclut des externalités sur leur environnement naturel et social, autrement dit sur leur territoire. Elles décident en effet, de l'allocation des ressources utilisées pour produire, notamment en choisissant de mobiliser plus ou moins de travail ou de capital, ou encore en utilisant telle ou telle matière première. Elles impactent aussi leur environnement social par la distribution qu'elles font de la richesse créée. Cette dernière est d'une part monétaire et d'autre part sociale dans le sens où non seulement les entreprises peuvent générer des liens sociaux, du capital social, mais aussi où elles sont susceptible de participer au bien-être des populations, le travail étant un pilier de la construction personnelle des individus.

Le choix est fait d'adhérer à cette conception du rôle des entreprises dans la société, il convient alors de considérer l'entreprise au sens large d'organisation de production d'un bien ou d'un service, indépendamment de son statut juridique et de son secteur d'activité. Autrement dit, dès lors qu'elles produisent un bien ou un service, les structures de l'économie sociale (associations, coopératives, mutuelles et fondations) et les administrations publiques sont incluses dans un ensemble appelé "les entreprises ». Cette vision met l'accent sur la fonction de production des entreprises, plutôt que sur leur fonction d'organisation des échanges marchands. 
Depuis quelques décennies, les mouvements liés à la Responsabilité Sociétale des Entreprises (RSE) et ceux liés au Développement Durable sont porteurs de création de richesses sociales par les entreprises, en incitant à l'innovation sociale qui en est le vecteur principal. Les entreprises actives en la matière intègrent, dans leurs activités, les interférences avec leur environnement, elles deviennent actrices d'un territoire qui reste à préciser. Leurs actions interfèrent avec celles des collectivités territoriales et l'étude des conditions de coordination doit être approfondie.

$\mathrm{Au}$ travers d'une analyse du contenu de 50 entretiens réalisés auprès d'entreprises socialement innovantes, il s'agit, dans cet article, d'une part de repérer l'utilisation de tel ou tel mot relatif au territoire et d'autre part d'analyser les définitions sous-jacentes. « $L a$ confiance est un facteur déterminant de la performance collective »(Prax, 2008, p. 26) et elle repose notamment sur le partage de valeurs exprimées au travers de mots entendus selon un sens commun.

La première partie de l'article présente et justifie les choix conceptuels et méthodologiques, en faveur d'une approche évolutionniste, pluridisciplinaire et ascendante (bottom up). L'innovation sociale apparaissant comme un processus comportant plusieurs étapes, et chaque étape laissant apparaître un vocable territorial particulier, ces phases sont ensuite successivement analysées.

\section{Une démarche évolutionniste, pluridisciplinaire et ascendante}

Étudier le langage, et en particulier sa capacité à constituer un outil d'échange et de coordination susceptible de favoriser l'action collective, suppose de se placer dans une perspective dynamique. En effet, quelles sont les causes de l'utilisation d'un mot plutôt qu'un autre? Quels en sont les effets? Telles sont les questions générales qui se posent et qui suggèrent dès le premier abord que, pour trouver des éléments de réponse, il va falloir regarder dans le temps, s'ouvrir au différents sens que peuvent prendre les mots et partir de l'observation du terrain. Cette posture est confirmée par la définition des notions sur lesquelles est centrée la recherche.

\subsection{L'innovation sociale est un processus}

D’une manière générale, l'innovation sociale vise l'amélioration de la qualité de vie (Cloutier, 2003). En fait, elle revêt de multiples facettes :

- « l'innovation sociale dans les entreprises pour favoriser l'amélioration de la productivité, elle se traduit par de nouveaux rapports entre les entreprises, entre les dirigeants et les dirigés ;

- l'innovation sociale pour améliorer la qualité de vie, elle consiste à créer de nouveaux dispositifs, à assurer de nouveaux services ;

- l'innovation sociale dans une perspective d'apprentissage collectif, d'accumulation de capital cognitif, elle se concrétise par des modalités plus démocratiques de développement et de gouvernance. » (Klein, Harrisson, 2007, p. 3).

Cette définition proposée par les chercheurs du CRISES (Centre de Recherche sur les Innovations Sociales, Université du Québec à Montréal, Canada) relie ouvertement le premier champ aux entreprises, sans le faire pour les deux seconds. Pourtant, les 
entreprises y sont aussi présentes ; elles y rejoignent l'ensemble des acteurs des territoires. Les analyses du rôle de l'innovation sociale sur le développement territorial, qui constituent un domaine de recherches et surtout de recherches-actions à part entière (Hillier, Moulaert, Nussbaumer, 2004), montrent comment l'innovation sociale résulte d'une construction dynamique associant divers acteurs dont les entreprises.

Plutôt que de considérer l'innovation sociale comme un objet finalisé classé dans telle ou telle catégorie, ce qui peut, en outre, conduire à fermer le champs de la créativité qui par définition ne peut pas l'être, le choix est fait ici, dans une optique dynamique et évolutionniste, de voir l'innovation sociale comme un processus dont les étapes successives ont chacune leurs caractéristiques propres. Les trois principales étapes sont :

- l'émergence de l'idée ;

- la mise en œuvre, la concrétisation ;

- la diffusion de l'innovation.

Par analogie avec l'innovation technologique, les deux premières phases peuvent être respectivement rapprochées de l'étape de "recherche », débouchant sur une invention, et de l'étape de «développement», débouchant sur une innovation. Si l'on retrouve a priori une correspondance entre innovation technologique et innovation sociale pour les deux premiers stades de la $R \& D$, la troisième phase de diffusion est différenciée. Alors que la richesse économique générée par l'innovation technologique est généralement perçue comme une rente revenant individuellement à l'innovateur, il n'en va pas de même pour l'innovation sociale, créatrice de richesse sociale, dont la vocation est d'être collectivement partagée, diffusée.

En fait, chacune des trois étapes est elle-même un processus évolutionniste de construction.

- Une idée émerge au fur et à mesure qu'un individu, ou un groupe d'individus, l'imagine puis la formule et la questionne dans le but de rechercher un maximum de pertinence par rapport à une situation donnée.

- La mise en œuvre de l'idée consiste à surmonter des obstacles et saisir des opportunités. Le passage à l'acte nécessitant souvent divers moyens et compétences, le processus consiste à fédérer un collectif autour du développement et de la maturation du projet innovant.

- La diffusion a pour but l'acceptation, la reconnaissance voire la duplication de l'innovation sociale. L'adaptation à d'autres individus, à d'autres situations, est le fil conducteur du processus.

Le langage partagé est au cœur de chacun des trois sous-processus, pour formuler, fédérer et adapter l'innovation sociale. Il est aussi une "clé » pour passer d'une étape à l'autre puisqu'il est nécessaire pour expliquer, éduquer et convaincre.

L'hypothèse proposée est qu'à chacune des étapes sont associée des acteurs impliqués particuliers ; à chacune des étapes est associée une relation particulière au territoire ; à chacune des étapes correspond un langage spécifique. "Le leadership qui intervient dans la réussite des initiatives locales se présentes sous trois formes : individuelle, organisationnelle et socio-territoriale »(Klein, 2011, p. 182). La question du territoire interviendrait alors comme élément de contexte environnant pour l'émergence de l'idée, comme facteur favorisant ou contraignant pour la mise en œuvre et comme partie-prenante, par le biais de ses représentants, de la diffusion. Quant au langage, aucune hypothèse n'est faite, il constitue le domaine à explorer. 


\subsection{Le territoire : une notion et un objet vivants}

Le territoire est avant tout un espace géré par des élus et des équipes administratives mais bien d'autres conceptions existent et les multiples dimensions du territoire, comme pour l'innovation sociale, ne facilitent pas sa définition ${ }^{1}$. Les conceptualisations sont nombreuses. Les économistes vont plutôt envisager le territoire comme un espace géographique qui peut être caractérisé par des ressources plus ou moins mobiles, mais aussi par des spécificités sociales de type identitaire, des règles et des conventions partagées (Requier-Desjardins, 2009). Les géographes vont plutôt considérer que le territoire se définit comme un ensemble de relations entre une population et un espace. Parmi les sociologues, le territoire est souvent approché comme un réseau.

Outre ces approches marquées disciplinairement, les courants pluridisciplinaires s’intéressent eux aussi à la question. En particulier, le courant de l'économie géographique intègre à l'analyse d'un territoire l'étude des choix et stratégies des populations qui raisonnent en termes de proximités. Les dimensions de cette proximité sont géographiques, organisationnelles et institutionnelles (Pecqueur, 1992) et font respectivement référence à ce qui ne peut être qu'un sentiment d'appartenance à un même espace géographique, une même organisation ou une même communauté de pensées et d'idées. Dans les démarches rattachées à la sociologie économique, des analyses systémiques sont développées (Itçaina, Palard, Ségas, 2007).

Loin d'être contradictoires, ces différentes définitions et approches du territoire se complètent et font la richesse de la notion ; en évoluant dans le temps elles le rendent vivant et lui confèrent une grande portée heuristique (Vanier, 2009). Toutefois, si les théoriciens utilisent des vocables très diversifiés pour évoquer le territoire, il en va de même, sur le terrain, pour les acteurs du territoire. Ce dernier peut alors devenir une source de confusion, voire de mésentente, quand les mots et les sens qui s'y rapportent ne sont ni entendus, ni partagés. Alors même que les acteurs peuvent poursuivre des objectifs communs, la dynamique territoriale peut en être entravée.

La question du développement du territoire est essentielle du fait que «les territoires se renouvellent dans leurs formes et leurs contenus »(Proulx, 2008, p.8). Ce développement territorial ou local, selon l'échelle choisie de la plus large à la plus réduite, intègre à la fois la préservation et le développement d'un patrimoine social et naturel. Tel est l'objectif de toutes les collectivités de gestion et d'animation des territoires. Les populations, à titre individuel ou collectivement, peuvent être aussi intéressées et/ou actives. En effet, la créativité et l'innovation résultent autant de démarches top down que d'initiatives bottom up (Godet, Durance, Mousli, 2010), elles sont au cœur de ce que l'on peut nommer l'innovation territoriale.

\subsection{Quand innovation sociale et innovation territoriale se confondent}

Dans un contexte de mondialisation sociale et de globalisation économique, la résurgence de préoccupations territoriales, parfois très localisées, revêt de nouveaux enjeux se traduisant par de nombreuses innovations territoriales. Celles-ci s'inscrivent dans deux axes

\footnotetext{
${ }^{1}$ Les deux dictionnaires de géographie consultés, Les mots de la géographie : dictionnaire critique (Brunet R., Ferras R., Théry H. (dir.), 2005) et Dictionnaire de la géographie et de l'espace des sociétés (Lévy J., Lussault M. (dir.), 2003) recensent respectivement six et huit sens différents pour définir le territoire.
} 
principaux : d'une part la création de nouveaux vecteurs d'identification à un territoire et d'autre part le renforcement de l'attractivité des territoires par la différenciation positive. Ces deux axes peuvent tout à fait se rejoindre mais fondamentalement, ils sont respectivement liés à l'implication citoyenne dans les projets de territoire pour le premier axe, et aux progrès en matière de qualité de vie pour le second. Au vue des conceptions du rôle social de l'entreprise et de l'innovation sociale telles qu'elles ont été énoncées précédemment, le lien apparaît clairement : les entreprises en inventant de nouvelles formes de gouvernance, de nouvelles formes de coordinations partenariales et en créant de la richesse sociale participent à l'innovation territoriale qui est à la base du développement territorial.

L'innovation sociale est d'une part territorialisée et d'autre part territorialisante (Fontan, 2011). En effet, premièrement, comme tout autre changement, elle est issue, à l'origine, soit d'une crise à laquelle il faut trouver une solution, soit d'une utopie qu' un ou plusieurs individus décident de concrétiser. Le contexte territorial, autrement dit l'environnement global des individus fait partie des facteurs déterminant de leurs aspirations, de leurs rêves, de leurs idées d'innovation sociale. Deuxièmement, quand les individus passent à l'action, l'innovation sociale crée du social, des liens, elle change les relations entre les personnes, entre les personnes et leur environnement ; elle participe ainsi à une forme de territorialisation.

Si le langage est un facteur de la réussite des processus d'innovation sociale, il l'est aussi pour la réussite des processus d'innovation territoriale. L'étude des nouvelles formes de gouvernances territoriales inventées dans le cadre du traitement des questions environnementales en est une bonne illustration. Elle montre qu'un grand nombre d'obstacles, de paradoxes, compromettent la réussite des procédures démocratiques de consultation mises en place. En résumé, ces difficultés concernent d'une part le problème de «penser global et agir local » et d'autre part l'instauration de conditions propices au dialogue constructif entre les experts et les citoyens (Theys, 2003). Sans être une solution ni miracle, ni unique, l'établissement d'un langage commun constitue sans aucun doute une clé favorisant le passage de la pensée à l'action et le support de base pour l'échange entre acteurs diversifiés. L'écoute, la compréhension, le compromis et la prise de décision collective passent par le langage.

\section{Une recherche appliquée au cas de la Basse-Normandie, France}

La Basse-Normandie est une Région du nord-ouest de la France qui conserve un caractère rural très marqué. Elle est aussi caractérisée par un secteur industriel encore très présent, au dessus de la moyenne nationale, malgré le phénomène de désindustrialisation en cours. Les tendances démographiques sont à peu près les mêmes qu'au niveau national : accroissement naturel positif et vieillissement de la population.

Dans le cadre d'un programme de recherche financé par le Conseil Régional, des entretiens ont été réalisés au début de l'année 2009 sur le thème de l'innovation sociale dans les entreprises.

\subsection{Les «mots » du territoire}

Cinquante entreprises ont été désignées comme socialement innovantes par des responsables de structures institutionnelles représentatives de différents types d'entreprises et de différents secteurs d'activité (Chambre Régionale des Métiers et de 
l'Artisanat de Basse-Normandie, Chambre Régionale de l'Économie Sociale et Solidaire, Union Régionale des Sociétés Coopératives et Participatives, Chambre Régionale de Commerce et d'Industrie, Conseil Régional de Basse-Normandie).

La démarche de recherche étant illustrative, la question de la représentativité de l'échantillon ne se pose pas. De fait, les entreprises relevant de l'Economie Sociale et Solidaire, soit de par leur statut juridique, soit de par leur activité principale (insertion, commerce équitable, services à la personne...) sont très nettement majoritaires, ce qui conforte les analyses selon lesquelles les entreprises de l'économie sociale sont par «nature » porteuses d'innovation sociale (Lévesque, 2004). Les autres entreprises sont de statut privé, ou public et elles ont été repérées pour des innovations sociales telles que la mise en œuvre de labellisations Développement Durable, l'expérimentation de procédures de Responsabilité Sociétale des Entreprises (RSE) ou suite à des opérations de marketing social/affinitaire.

La thématique des entretiens était l'innovation sociale. Le guide d'entretien ne comportait donc pas de questions particulières sur le territoire, sauf une sur la localisation des clients, fournisseurs et partenaires de l'entreprise, au niveau de l'identification. Les personnes rencontrées se sont donc spontanément exprimées sur ce thème du territoire, ce qui démontre, conformément aux hypothèses, de l'existence d'une forte inférence. De plus, il n'est pas apparu de distinction entre les entreprises de statut différent quant à l'utilisation des mots concernant le territoire.

L'analyse de contenu consiste à examiner les informations clairement énoncées mais aussi les indicateurs de style et de comportement susceptibles de délivrer d'autres indices. Les méthodes thématiques et fréquentielles restent néanmoins les plus aisées, les plus fiables et les plus utiles, dans un premier temps, pour la majorité des matériaux (Bardin, 2001). L'analyse est donc ici sémantique, elle porte sur les mots clés habituellement associés au territoire. Ces mots sont : « territoire », « local », « proximité » et « développement ». Dans les entretiens, ils ont été utilisés avec des sens différents selon que les responsables d'entreprises expliquaient la phase d'émergence de l'innovation sociale, l'étape de concrétisation du projet ou sa diffusion.

Outre la recherche du sens, l'analyse lexicale consiste aussi à compter les mots. La recherche des récurrences a servi de base à l'étude des occurrences qui finalement s'est avérée plus riche d'enseignements que celle des récurrences. En effet, d'une part, le guide d'entretien n'était pas globalement structuré autour des trois étapes de l'innovation sociale, l'émergence, la réalisation et la diffusion ${ }^{2}$, et de nombreuses questions étaient ouvertes. D'autre part, l'utilisation des mots relève parfois d'expressions conventionnelles, les personnes y recourant sans vraiment leur donner de sens et il n'est alors pas très probant de les compter ${ }^{3}$. En particulier, le mot «local » se retrouve fréquemment cité pour désigner la «mission locale », le «plan local », le «comité

\footnotetext{
${ }^{2}$ Le guide d'entretien semi-directif était structuré, dans une optique didactique, autour de la réflexion sur ce qu'est une innovation, ce qu'est le social, sur le rôle social des entreprises et pour finir sur l'innovation sociale.

${ }^{3}$ C'est le cas pour les mots « région » (« régional »), « département » (« départemental », « conseil général »), « commune » (« ville ») dont le sens ne présente pas d'ambiguïté. Ils sont utilisés quasiment exclusivement pour désigner les instances politiques d'administration territoriale. Dans quelques rares cas, le mot « région » est utilisé dans l'expression « changer de région » pour évoquer une relocalisation dans l'espace d'un individu ou d'une entreprise.
} 
local », la « collectivité locale »... Mises à part ces récurrences particulières, le constat sur l'ensemble des entretiens est que les mots «local » et «proximité » sont ceux qui reviennent le plus souvent, avec environ 100 énonciations chacun. Ont aussi été comptés les mots du même radical, respectivement : «localement », «localisation », " délocaliser », « relocaliser » et « proche », « rapprocher », « rapprochement», « approche ». Arrivent ensuite à égalité en seconde place, avec environ 70 énonciations chacun, les mots «territoire » (et «territorial») et « développement ». Deux cas apparaissent suffisamment particuliers pour mériter un traitement spécial : le verbe « développer » utilisé 60 fois à peu près et le «développement durable » répété 140 fois $^{4}$ !

\subsection{L'émergence de l'innovation sociale : le territoire de l'individu}

Les propos recueillis auprès des responsables d'entreprise rencontrés permettent de construire, non pas une définition supplémentaire, mais une sémantique particulière du territoire correspondant à l'apparition de l'idée et au démarrage du projet d'innovation sociale. Les personnes ont été interrogées sur leur conception théorique de l'innovation, du processus de création, sur leur parcours individuel, leur réflexion, le sens de leur démarche, sur les faits relatifs à l'émergence de l'idée, les facteurs qui leur sont apparus favorables et les freins.

Il semble que le point de départ de l'innovation sociale corresponde en général à deux situations types : soit une prise de conscience qui intervient dans le cadre d'une réflexion générale sur la société, soit une exclusion du système économique conventionnel, qu'il s'agisse d'une faillite d'entreprise, d'un licenciement, d'un état de pauvreté. La première situation pourrait a priori être positive, contrairement à la seconde, mais les propos tenus lors des entretiens conduisent à relativiser. Dans les deux cas, l'innovation sociale "relève d'aspirations à créer des milieux de vie responsables, où se résorbent certains effets néfastes de la pauvreté et où se retissent des liens sociaux » (Bouchard, 2006) ; la posture des innovateurs est combative.

Pour exprimer ce qui les a poussés vers l'innovation sociale, les personnes entretenues qui utilisent le terme "territoire $»^{5}$, le font en personnifiant le territoire : la "faiblesse des revenus du territoire », les "besoins du territoire », la nécessité " d'accompagner le territoire » sont autant de motivations évoquées. Les individus interprètent ce qu'ils observent autour d'eux, au travers de jugements qu'ils portent par rapport à des valeurs éthiques. Leur interprétation est très globale, elle porte sur la condition humaine et n'a pas de frontière explicitée.

L'élément déclencheur de l'émergence d'une idée est quant à lui plus cerné, ce qui apparaît en recherchant le mot "proximité », ainsi que les mots du même radical. En théorie, "le local renvoie à l'échelle tandis que le territorial renvoie au mode de construction par les acteurs, quelle que soit l'échelle »(Pecqueur, 2009, p.57). Effectivement, sur le terrain, l'idée exprimée est de « se rapprocher des problèmes pour se rapprocher des solutions » : «l'observation du tissu local» suscite « des initiatives locales ». Ce rapprochement

\footnotetext{
${ }^{4}$ Le record est détenu par une personne qui à elle seule emploie l'expression « développement durable » 38 fois en 1 h30 d'entretien.

${ }^{5}$ Personne n'utilise les mots « territorialité » ou « territorialisation ».
} 
est physiquement marqué dans l'espace géographique, la plupart du temps à l'échelle du quartier et du voisinage.

Le concept de territoire prend ici une dimension subjective à deux facettes : le territoire interprété et la proximité de situation qui lui donnent une dimension proxémique telle que l'envisagent les géographes mais dans une perspective très humanisée et personnalisée. Les mots liés au territoire s'insèrent dans un discours éthique ${ }^{6}$, propre à chacun, basé sur l'argumentation plutôt que sur l'affirmation où "l'ultime preuve de la réussite de l'argumentaire est l'action » (Koren, 2008, p.14).

L'idée de l'innovation sociale se révèle donc en réaction au contexte environnant. Depuis longtemps il est tenu pour acquis, comme Polanyi le montre si bien dans La Grande Transformation, le fait que les crises, les milieux défavorisant, vont forcer la volonté à agir et provoquer l'innovation, quand la liberté d'action des individus le permet bien sûr. Les théories supposant l'existence de milieux propices, comme la théorie des systèmes d'innovation, apparaissent plus adaptée à l'innovation technologique $\mathrm{du}$ fait qu'elles traitent la question sociale en tant qu'externalités du développement économique et qu'elles laissent généralement de côté les entreprises publiques et les entreprises de l'économie sociale (Lévesque, 2008, p.56). De même, les théories des milieux innovateurs ne semblent pas non plus adaptées, étant trop positives quant aux incitations supposées. Les acteurs de l'innovation sociale rencontrés lors des entretiens se placent, pour beaucoup, effectivement dans une posture transgressive. Ils préconisent « d'autres approches » de l'entreprise, de «nouvelles approches » qui mettraient en cohérence leurs valeurs éthiques et l'entreprise, "des approches globales », "des approches inversées ». Ces expressions suggèrent une sorte d'anti-proximité qui résulte de la réflexion et du positionnement éthique, et qui viendrait s'ajouter à la proximité de situation évoquée ci-dessus.

Finalement, cela débouche sur une mise en situation d'opposition locale. La posture est même, dans quelques cas, celle d'une opposition très affirmée. Le constat est clair quant il s'agit d'observer et de comprendre l'occurrence et la récurrence des mots « délocalisation » et « relocalisation ». En démontrent les appels à « se prendre en charge », "à se prendre en main », "à réparer les dégâts par l'innovation sociale », "à devenir acteur de l'économie locale ». Les innovateurs ont là, en réaction, des idées d'innovation sociale très ancrées spatialement ; un espace «local de contestation» apparaît (Lévesque, 1999, p.5).

L'expression " développement durable » est massivement présente dans les retranscriptions d'entretien pour ce qui concerne cette étape d'émergence. Beaucoup se placent, là encore, en opposition par rapport aux entreprises qui communiquent sur le développement durable sans réellement agir. Le développement durable correspond à des visions éthiques du monde qui incitent à l'innovation sociale. De nombreuses personnes interrogées « y croient » et sont souvent déçus par ce qui en est effectivement fait. Le développement durable est "un champ variable de réflexions, de pratiques et de prises de conscience, bien davantage qu'une réalité univoque » (Levy, Lussault, 2003, p.249). De fait, le développement durable n'est pas un concept scientifique facile à définir, il comporte une dimension éthique forte et inclus donc un jugement normatif. Au regard de

\footnotetext{
${ }^{6}$ La forme discursive doit être considérée par rapport à la situation de communication (Charaudeau, Maingueneau, 2002). Ici le cadre est la réalisation d'entretiens de recherche universitaire.
} 
l'utilisation qui est en est faite dans les entretiens étudiés, le développement durable est évoqué pour désigner le référentiel de construction du territoire interprété de l'individu.

En résumé sur cette première phase d'émergence de l'innovation sociale, au travers des mots qui lui sont associés, le territoire apparait comme très intériorisé par les individus, chacun en ayant une vision personnelle propre. Par contre, le processus est à chaque fois le même : le territoire interprété, la proximité de situation et l'éthique conduisent les porteurs d'idée à se positionner localement en opposition pour amorcer des solutions d'innovation sociale.

\subsection{La concrétisation de l'innovation sociale : le territoire de l'entreprise}

Lors des entretiens, les responsables d'entreprises se sont exprimés sur les caractéristiques de l'innovation sociale qu'ils ont développée, sur les conditions de mise en œuvre du projet, de sa structuration et sur leur appréciation du contexte de sa réalisation.

À ce stade, les individus, les groupes d'individus sont acteurs, ils influent sur le territoire et non l'inverse comme dans l'étape d'émergence. Le territoire est vécu (Di Méo, 1994), élaboré par les acteurs, Pecqueur (2009, p 56) parle du "territoire construit », Massicotte (2008, p. XV) évoque un «territoire d'action ». À ce stade le développement durable est, comme pour la phase d'émergence, une expression très usitée, la concrétisation de l'innovation sociale prend alors le sens d'une manière de "faire ensemble autrement » : "faire du développement durable », "prendre le virage du développement durable », "s'engager dans le développement durable ». Le territoire relève alors du " domaine des pratiques de tous ordres qui démarquent spatialement tel groupe social par rapport à tel autre » (R. Hérin cité dans Vanier, 2009, p. 84).

La proximité est considérée comme une ressource pour développer l'activité, tel un véritable facteur de production. À ce stade, les questions de communication sont centrales car "le langage représente une matière première indispensable pour assurer la coopération entre acteurs au travail » (Charaudeau, Maingueneau, 2002, p.586). Au sens où la proximité crée des liens sociaux, où elle permet la communication et facilite la transmission d'informations, elle est un gage de transparence qui « rend les choses plus simples ». Le langage est l'outil principal de mise en œuvre de la proximité ; il permet une production de connaissance qui va accompagner et fonder la décision, c'est-à-dire l'action (Amelot, André-Lamat, 2010). En ce sens, l'identification de «la demande de proximité » est le moyen de pouvoir répondre à des besoins réels, ce qui est une aspiration forte de nombreux responsables d'entreprises rencontrés. Ces démarches de proximité sont un vecteur important d'innovation sociale en correspondance avec les « cinq principes pour réussir à mener un projet collectivement : l'exigence d'inventaire des groupes concernés et de leurs problèmes, l'exigence d'expression surtout pour les groupes émergents qu'il faut intégrer dans le collectif, l'exigence d'exploration pour aider à préciser les problèmes, l'exigence de composition pour faciliter l'expression des demandes et l'exigence d'itération » (Callon dans Klein, Harrisson, 2007, p39-40). Cette proximité est mise en œuvre vis-à-vis des clients de l'entreprise mais aussi vis-à-vis des salariés. Il ne s'agit plus d'être "proche du patron pour être entendu », il s'agit, pour l'entreprise, d'être «au plus proche des besoins des salariés » par le biais de temps d'échanges et de dispositifs cognitifs d'écoute mutuelle. L'entreprise est ainsi actrice du bien-être des individus qu'elle met en réseau, qu'elle intègre dans une communauté. 
Pour aller plus loin dans l'analyse et déboucher sur le concept de proximité organisationnelle, il convient de remarquer que la démarche de création et d'entretien de liens sociaux est aussi appliquée vis-à-vis des autres acteurs de la vie locale. Il s'agit alors de " travailler avec des producteurs locaux », "des entreprises locales », « des fournisseurs locaux » et même «des banques locales ». Les collectivités ne sont pas les seules à être locales, les partenaires de l'entreprise acquièrent aussi cette qualité.

Pendant cette étape de réalisation de l'innovation sociale, le territoire devient donc collectif. L'entreprise élabore un réseau social et déclenche ainsi un processus de territorialisation. Le territoire de l'entreprise n'est ni le marché, ni la zone de chalandise qui apparaissent comme des champs de vision beaucoup trop restrictifs. Avec l'innovation sociale, le territoire de l'entreprise n'est plus seulement économique, il devient aussi géographique, politique et social. Sur le plan spatial, le territoire de l'entreprise se situe à l'échelle des communes environnantes, de la ville, du quartier, le voisinage étant même cité par la plus grande entreprise enquêtée. Cette dernière est présente sur les marchés internationaux mais son «territoire est local ». Le potentiel de proximité géographique est activé et mobilisé (Torre, 2009).

Si les mots «proximité » et «local » sont utilisés dans un sens qui démontre clairement l'existence et les caractéristiques d'un territoire de l'entreprise, il n'en va pas de même pour le mot «développement». Quant il ne s'agit pas du développement durable, les interviewés recourent à ce mot, et aux mots du même radical, surtout le verbe "développer », pour faire référence au développement économique stricto sensu : "développement de l'entreprise », "développement d'une activité », "projet de développement », « recherche et développement». Le « développement local » n'est pas évoqué. Le développement social est avancé comme une condition du développement économique et une seule fois quelqu'un parle de « développement personnel ». La dimension économique reprend donc parfois le dessus et plusieurs personnes racontent avec regrets comment, malgré eux, s'instaure une concurrence entre les territoires d'entreprise : "on essaie de ne pas se marcher sur les plates-bandes et de travailler ensemble sur nos territoires ». Quand ces interviewés racontent la mise en œuvre de l'innovation sociale le mot «territoire » revêt une dimension de possession très affirmée, allant même jusqu'à une connotation guerrière : «c'est mon territoire », pour dire «mon marché, ma clientèle », "ils empiètent sur mon territoire », "on vient marcher sur mon territoire ». En quête "d'identité territoriale », les territoires d'entreprise construits autour de l'innovation sociale ne s'ouvrent pas dans un premier temps aux territoires extérieurs, c'est-à-dire aux autres entreprises, aux autres secteurs, aux autres régions. Ils cherchent même à s'en tenir à distance.

En résumé, pour la phase de réalisation de l'innovation sociale, chaque groupe d'individus, autrement dit, chaque entreprise, construit, édifie et s'approprie son propre territoire, son propre réseau, autant spatialement, en «travaillant avec les communes environnantes » par exemple, que socialement, en «travaillant pour les gens du quartier » par exemple. À l'intérieur du territoire de l'entreprise, les efforts de construction d'un langage partagé sont importants, ils sont une condition nécessaire à l'identité du territoire en question. Le « local » et « la proximité » sont les mots cardinaux. Au contraire, vis-à-vis de l'extérieur, la logique économique est très prégnante et instaure une concurrence territoriale peu propice au dialogue. Les mots « développement» et «territoire » sont emblématiques de cette situation. La recherche d'affinités identitaires extérieures à l'entreprise-réseau arrive dans une étape ultérieure, celle de la diffusion de l'innovation sociale. 


\subsection{La diffusion de l'innovation sociale : le territoire politique}

L'étude de la diffusion, un aspect essentiel de l'institutionnalisation, vient clore l'analyse du processus d'innovation sociale. La diffusion est entendue comme le passage de l'expérimentation pilote à la multiplication d'expériences. Sur ce point, les responsables d'entreprise se sont exprimés sur la manière dont leur démarche d'innovation sociale a été comprise, ou pas, sur la reconnaissance de leurs actions et sur leurs perspectives et projets suite à l'innovation sociale mise en œuvre.

Le fait que le processus de diffusion de l'innovation sociale à d'autres entreprises est radicalement différent de celui de l'innovation économique ${ }^{7}$ se trouve effectivement vérifié. Alors que, pour profiter de la rente de monopole, l'entrepreneur à l'origine d'une innovation économique n'est pas enclin à la diffusion de celle-ci, l'innovation sociale qui est vue, non pas comme une source de revenu monétaire, mais comme la réalisation d'un projet de société, a vocation à être diffusée. Une personne interrogée déclare "j'agis sur les gens qui me sont proches, à charge pour eux d'agir sur d'autres ». L'innovation sociale est donc bien fortement ancrée localement, la démonstration en est de ce point de vue enrichie. Dans le même temps, du fait qu'elle est basée sur l'échange et le dialogue, elle est intrinsèquement appelée à être multipliée, appliquée à d'autres situations locales. Son expérimentation à un endroit favorise son implantation ailleurs. Durant les entretiens, une définition de l'innovation sociale est revenue à plusieurs reprises la présentant comme une pratique d'entreprise qui ne "se faisait pas localement, mais qui ne venait pas de nulle part ». L'échange de pratiques, les «laboratoires d'idées » sont des moyens sur lesquels s'appuie la diffusion de l'innovation sociale. Elle participe à l'élargissement du territoire de l'entreprise.

Cette diffusion vise d'autres acteurs, par exemple, les entreprises de l'Économie Sociale et Solidaire s'interrogent sur la diffusion de l'innovation sociale au sein du «business classique ». Elle vise aussi d' autres territoires au sens des autres administrateurs, aménageurs du territoire, principalement les Régions, Départements et Pays. Le territoire n'est plus local comme pour l'étape précédente, il est global tout en restant «à une taille humaine ».

Le « territoire » est le mot le plus fréquemment associé aux questions liées à la diffusion. Il est utilisé et entendu au sens de la région, souvent la région administrative. Il s'agit alors " d'aborder les territoires », "de construire une relation avec le territoire », " de travailler avec le territoire », "d'occuper les territoires », "d'animer les territoires », "d'interpeler d'autres territoires», de "s'allier avec les territoires ». Si l'expression « aménagement du territoire » n'est énoncée que deux fois sur l'ensemble des entretiens, elle l'est pour discuter de la diffusion de l'innovation sociale.

Pour la plupart des interviewés, les collectivités territoriales ont donc un rôle-clé à jouer pour la diffusion des innovations sociales. Et pas seulement sur un plan financier puisqu'il s'agit aussi d'instaurer un «dialogue entre les territoires », entre les acteurs. Cette position d'intermédiaire entre les acteurs au sein d'un même territoire, et entre différents niveaux de

7 Selon la typologie, conventionnellement admise, issue des travaux de Schumpeter, l'innovation économique peut prendre cinq formes : l'innovation de produit, de procédé, organisationnelle, de marché et de matières premières. L'innovation est économique quand on adopte un point de vue de gestionnaire de la rentabilité de l'entreprise, elle est sociale quand on adopte un point de vue lié à la société. Toute innovation revêt en fait les deux aspects. 
territorialité est stratégique pour faire valoir les différences de pratique entrepreneuriale en termes d'exemplarités : " grâce à la Région, on a rayonné dans d'autres régions » affirme l'un des innovateurs rencontrés. Ce qui compte, "c'est la diffusion hors du territoire » dit un autre. La reconnaissance médiatique est aussi essentielle. S'il est courant d'entendre parler des entreprises subventionnées qui se délocalisent, les entreprises subventionnées «qui ont à cour d'avoir un rôle sur le territoire » sont peu mises en avant. Plusieurs personnes ont tenu implicitement ou explicitement ce discours pendant les entretiens.

Dans une optique dynamique, le territoire existe dès lors qu'il se construit un patrimoine. Le « développement durable » est une expression centrale dans les discours fédérateurs pour une patrimonialisation du territoire : il faut "développer les réseaux pour le développement durable », " regrouper les élus autour du développement durable ", "renforcer les associations pour le développement durable ", " diffuser les labels développement durable ». L'innovation sociale devient alors fondatrice de l'identité territoriale des entreprises. Elle représente aussi un potentiel intéressant pour la construction de l'identité d'un territoire plus global que local. Certaines collectivités territoriales l'ont bien compris comme les innovateurs interrogés. "L'adoption d'une stratégie de développement durable pourrait conduire à l'épanouissement non seulement de l'économie sociale et solidaire, mais aussi d'une économie plurielle, y compris pour l'ensemble de l'économie » (Lévesque, 2008, p. 226) D'où un intérêt commun à l'organisation de manifestations et d'actions publiques autour du développement durable. À ces occasions, les discours sont entendus, les mots prennent sens, et même si les divergences d'interprétation subsistent, l'émergence d'innovation sociale est favorisée.

En résumé sur cette étape, la diffusion et l'institutionnalisation de l'innovation sociale donne lieu au renforcement d'une territorialité plus globale, qu'il est pertinent d'aborder en terme de proximité institutionnelle. La notion de territoire rejoint ici les espaces de gestion politiquement et administrativement institués, le «développement durable » en est l'expression cardinale.

\section{Conclusion}

La phase d'émergence n'est pas et ne peut pas être associée à des définitions habituelles du territoire. L'approche sémantique apporte les éléments montrant que le développement durable est force d'incitation et de motivation justement parce qu'il permet de multiples interprétations, une grande diversité de "visions $d u$ monde ». Dans cette approche très globale du territoire, à la fois sociale et environnementale, on retrouve une " part du rêve » déjà évoquée par Schumpeter pour caractériser l'état d'esprit de l'entrepreneur innovateur. Pour l'innovation sociale, le territoire rêvé correspond à une vision éthique du monde. La multiplicité des sens attribués aux mots et la posture transgressive des innovateurs ne facilitent pas la mise en place d'un langage commun.

L'étape de réalisation du projet, est quant à elle cohérente avec les concepts de territorialisation et de proximité organisationnelle. Le sens des mots est plus partagé mais les postures d'acteurs restent contrastées du fait qu'elles sont à la fois vouées à la construction collective d'un territoire interne à l'entreprise et dans le même temps en concurrence sur un territoire externe. Le langage commun est plus facile à mettre en place que dans la phase d'émergence, mais moins que dans la phase de diffusion. 
Cette dernière étape de la diffusion de l'innovation sociale est caractérisée par le partage des mots et des sens et par la convergence des postures. C'est la territorialité instituée. En particulier, les entreprises socialement innovantes et les structures publiques parviennent à trouver un terrain d'entente, un langage commun, pour développer l'innovation sociale non plus seulement au niveau de l'entreprise mais au niveau du territoire.

Le développement durable est moins un concept scientifique, qu'une pratique qui consiste à construire des territoires individuels et collectifs. Sur la base des entretiens étudiés, il est possible de penser que le développement durable a remplacé le «développement local $»^{8}$ dans la terminologie courante, sans que pour autant le rattachement à un lieu, un espace ait disparu.

Les particularités territoriales, en l'occurrence le caractère rural et les particularités historico-politico-socio-économiques de la Région étudiée, ont forcément une influence sur le développement régional et l'innovation sociale (Guesnier, Joyal, 2004) impactant ainsi les faits relatés et donc les analyses qui peuvent en être faites. Cependant, le recours spontanément massif aux notions de territoire dans les propos tenus démontre de l'importance des jugements personnels intellectuellement réfléchis, fondés et rigoureusement argumentés comme déterminants de l'action. Ces argumentations viennent étayer et enrichir les concepts théoriques.

Il est nécessaire de connaître les niveaux de capacité, de capabilitie, des personnes pour faire du développement socialement durable (Ballet, Dubois, Mahieu, 2004), le langage partagé apparaît comme une ressource essentielle pour y parvenir. Certes, «les mots ne font pas tout ", le « discours n'est pas l'action » mais pour favoriser l'innovation sociale et le développement des territoires, l'étude des mots et des discours se montre particulièrement constructive et instructive, en particulier pour les institutions politico-administratives et de gouvernance qui voudraient constituer « des catalyseurs de l'innovation sociale » (Hillier, Moulaert, Nussbaumer, 2004, p.146). De ce point de vue, des pistes pour l'action des pouvoirs publics sont ouvertes pour faciliter l'acceptation du changement et le progrès social.

\footnotetext{
${ }^{8}$ Sur l'ensemble des entretiens, le « développement local » n'a été cité que 4 fois, contre 140 pour le « développement durable».
} 


\section{Références}

Amelot X., André-Lamat V., 2010. «Faire » du développement durable au Sud. Production scientifique, construction du discours, déterminants de l'action. In Bouquet C. (dir.), Les géographes et le développement - Discours et actions. Maison des Sciences de l'Homme d'Aquitaine, Pessac, 89-104.

Ballet J., Dubois J-L., Mahieu F-R., 2004. À la recherche du développement socialement durable: concepts fondamentaux et principes de base. Développement durable et territoires, Dossier 3 : Les dimensions humaine et sociale du Développement Durable.

Bardin L., 2001. L'analyse de contenu. Presses Universitaires de France, Paris.

Bouchard M., 2006. De l'expérimentation à l'institutionnalisation positive : l'innovation sociale dans le logement communautaire au Québec. Annals of Public and Cooperative Economics 77 (2), 139-166.

Brunet R., Ferras R., Théry H. (dir.), 2005. Les mots de la géographie : dictionnaire critique. $3^{\mathrm{e}}$ édition revue et augmentée, La Documentation française, Paris.

Charaudeau P., Maingueneau D. (dir.), 2002. Dictionnaire d'analyse du discours. Le Seuil, Paris.

Cloutier J., 2003. Qu'est-ce que l'innovation sociale ? Cahiers du CRISES, Collection études théoriques $\mathrm{n}^{\circ}$ ET0314, novembre.

Di Méo G., 1994. Patrimoine et territoire, une parenté conceptuelle. Espaces et Sociétés 78, 15-34.

Fontan J-M., 2011. Développement territorial et innovation sociale. In Bellemare G., Klein, J.L. (dir.), Innovation sociale et territoire. Convergences théoriques et pratiques, Presses de l'Université du Québec, Sainte-Foy, Canada, 17-42.

Godet M., Durance P., Mousli M., 2010. Créativité et innovation dans les territoires. Rapport du Conseil d'Analyse Économique, Direction de l'information légale et administrative, La Documentation française, Paris.

Guesnier B., Joyal A. (dir.), 2004. Le développement territorial. Regards croisés sur la diversification et les stratégies. Datar (Délégation à l'Aménagement du Territoire et à l'Action Régionale) et IERF (Institut d'Économie Régionale et Financière), France.

Hillier J., Moulaert F., Nussbaumer J., 2004. Trois essais sur le rôle de l'innovation sociale dans le développement territorial. Géographie, Économie, Société 6 (2), 129-152.

Itçaina X, Palard J., Ségas S. (dir.), 2007. Régimes territoriaux et développement économique. Presses Universitaires de Rennes, Rennes.

Klein, J.L., 2011, Économie sociale et territoire en contexte de mondialisation. Le développement par l'initiative locale. In Bellemare G., Klein, J.L. (dir.), Innovation sociale et territoire. Convergences théoriques et pratiques, Presses de l'Université du Québec, Sainte-Foy, 175-194.

Klein, J.L., Harrisson D., 2006. L'innovation sociale, émergence et effets sur la transformation des sociétés. Introduction, Presses de l'Université du Québec, Sainte-Foy, 2-13.

Koren R., 2008. Pour une éthique du discours : prise de position et rationalité axiologique. Argumentation et analyse du discours 1.

Lévesque B., 2008. Contribution de la nouvelle sociologie économique à l'analyse des territoires sous l'angle de l'économie plurielle. Chapitre 7 In Massicotte G. (dir.), Sciences du territoire. Perspectives québécoises. Presses de 1'Université du Québec, Sainte-Foy, 205-232.

Lévesque B., 2007. L'innovation dans le développement économique et dans le développement social. In Klein J-L. et Harrisson D. (dir.), L'innovation sociale. Emergence et effets sur la transformation sociale. Presses de l'Université du Québec, Sainte-Foy, 43-70.

Lévesque B., 2004. Les entreprises d'économie sociale, plus porteuses d'innovations sociales que les autres ? In Le développement social au rythme de l'innovation. Presses de l’Université du Québec, Sainte-Foy, 51-72.

Lévesque B., 1999. Le développement local et l'économie sociale : deux éléments devenus incontournables du nouvel environnement. Cahiers du CRISES, Collection études théoriques, $\mathrm{n}^{\circ}$ ET9905, février.

Lévy J., Lussault M. (dir.), 2003. Dictionnaire de la géographie et de l'espace des sociétés. Belin, Paris. 
Massicotte G., 2008. Les sciences du territoire au Québec. Brève histoire de la construction d'un savoir. Avantpropos In Massicotte (dir.), Sciences du territoire. Perspectives québécoises. Presses de l'Université du Québec, Sainte-Foy, IX-XVIII.

Pecqueur B., 1992. Territoire, territorialité et développement. Actes du colloque industries et territoire : les systèmes productifs localisés. IREP-D Grenoble, octobre, 71-88.

Pecqueur B., 2009. De l'exténuation à la sublimation : la notion de territoire est-elle encore utile ? Géographie, Économie, Société 11 (1), 55-62

Prax J-Y., 2008. Réseaux sociaux, innovation et compétitivité : nouvelle donne pour la gouvernance territoriale. Les Cahiers du Management Territorial 34, 11-30.

Proulx M-U., 2008. Territoires de gestion et territoires d'émergence. Introduction In Guy Massicotte (dir.), Sciences du territoire. Perspectives québécoises. Presses de l’Université du Québec, Sainte-Foy, 1-19

Requier-Desjardins D., 2009. Territoires-Identités-Patrimoine : une approche économique ? Développement durable et territoires, Dossier 12 : Identités, patrimoines collectifs et développement soutenable, http:// developpementdurable.revues.org/index7852.html

Schumpeter J., 1939. Business Cycles: a Theoretical, Historical and Statistical Analysis of the Capitalism Process. McGraw-Hill, New York.

Torre A., 2009. Retour sur la notion de proximité géographique. Géographie, Économie, Société 11 (1), 63-75.

Vanier M. (dir.), 2009. Territoires, territorialité, territorialisation. Controverses et perspectives. Presses Universitaires de Rennes, Rennes. 